\title{
Mapping EORTC QLQ-C30 and FACT-G onto EQ-5D-5L index for patients with cancer
}

\author{
Yasuhiro Hagiwara ${ }^{* *}\left(\mathbb{D}\right.$, Takeru Shiroiwa $^{2}$, Naruto Taira ${ }^{3}$, Takuya Kawahara ${ }^{4}$, Keiko Konomura$^{2}$, Shinichi Noto ${ }^{5}$, \\ Takashi Fukuda² and Kojiro Shimozuma ${ }^{6}$
}

\begin{abstract}
Background: To develop direct and indirect (response) mapping algorithms from the European Organization for Research and Treatment of Cancer Quality of Life Questionnaire Core 30 (EORTC QLQ-C30) and the Functional Assessment of Cancer Therapy General (FACT-G) onto the EQ-5D-5L index.

Methods: We conducted the QOL-MAC study where EQ-5D-5L, EORTC QLQ-C30, and FACT-G were cross-sectionally evaluated in patients receiving drug treatment for solid tumors in Japan. We developed direct and indirect mapping algorithms using 7 regression methods. Direct mapping was based on the Japanese value set. We evaluated the predictive performances based on root mean squared error (RMSE), mean absolute error, and correlation between the observed and predicted EQ-5D-5L indexes.

Results: Based on data from 903 and 908 patients for EORTC QLQ-C30 and FACT-G, respectively, we recommend two-part beta regression for direct mapping and ordinal logistic regression for indirect mapping for both EORTC QLQ-C30 and FACT-G. Cross-validated RMSE were 0.101 in the two methods for EORTC QLQ-C30, whereas they were 0.121 in two-part beta regression and 0.120 in ordinal logistic regression for FACT-G. The mean EQ-5D-5L index and cumulative distribution function simulated from the recommended mapping algorithms generally matched with the observed ones except for very good health (both source measures) and poor health (only FACT-G).

Conclusions: The developed mapping algorithms can be used to generate the EQ-5D-5L index from EORTC QLQC30 or FACT-G in cost-effectiveness analyses, whose predictive performance would be similar to or better than those of previous algorithms.
\end{abstract}

Keywords: Cancer, EORTC QLQ-C30, EQ-5D-5L, FACT-G, Mapping, Preference-based measure

\section{Background}

Cancer is a common disease in many countries in the twenty-first century; there were estimated 18.1 million new cancer cases and 9.6 million cancer deaths in 2018 [1]. Although the advancements in cancer treatment prolong life and improve the quality of life of patients, the fight against cancer seems to have a long way to go. One recent problem related to cancer treatments is their cost.

*Correspondence: hagiwara@epistat.m.u-tokyo.ac.jp

${ }^{1}$ Department of Biostatistics, Division of Health Sciences and Nursing, The University of Tokyo, 7-3-1, Hongo, Bunkyo-ku, Tokyo 113-0033, Japan

Full list of author information is available at the end of the article
In the era of targeted, immune, and gene therapies, some treatments are highly effective but costly [2]. With limited medical resources, we need to evaluate not only the effectiveness of cancer treatments but also their costeffectiveness [3].

In cost-effectiveness analyses of cancer treatments, the most important and commonly used health outcome is quality-adjusted life year (QALY). QALY incorporates both the duration and quality of life, the two most important aspects for patients with cancer, and enables us to compare the cost-effectiveness of treatments in resource allocation irrespective of the disease area [4]. Calculation of QALY requires health utility data that are used as original author(s) and the source, provide a link to the Creative Commons licence, and indicate if changes were made. The images or other third party material in this article are included in the article's Creative Commons licence, unless indicated otherwise in a credit line to the material. If material is not included in the article's Creative Commons licence and your intended use is not permitted by statutory regulation or exceeds the permitted use, you will need to obtain permission directly from the copyright holder. To view a copy of this licence, visit http://creativecommons.org/licenses/by/4.0/. The Creative Commons Public Domain Dedication waiver (http://creativeco mmons.org/publicdomain/zero/1.0/) applies to the data made available in this article, unless otherwise stated in a credit line to the data. 
weights for quality of life. Since direct elicitation of health utility is burdensome, multi-attribute preference-based measures, such as EQ-5D are often used [5]. The National Institute for Health and Care Excellence in the United Kingdom prefers the use of EQ-5D in cost-effectiveness analyses [6], whereas the Center for Outcomes Research and Economic Evaluation for Health in Japan recommends a preference-based measure based on the time trade-off method [7, 8], which virtually indicates EQ-5D in the current situation. Since EQ-5D with 3 levels (EQ5D-3L) has several limitations, such as ceiling effect and multimodality $[9,10]$, EQ-5D with 5 levels (EQ-5D-5L) was developed and the value sets for it are now available in many countries [11].

Unfortunately, the EQ-5D data is often directly unavailable when cost-effectiveness analyses for cancer treatments are conducted. Instead, cancer-specific nonpreference-based health-related quality of life (HRQOL) measures that are directly relevant and sensitive to cancer-related treatments and symptoms are used to evaluate the HRQOL of patients with cancer. The two common cancer-specific HRQOL measures are the European Organization for Research and Treatment of Cancer Quality of Life Questionnaire Core 30 (EORTC QLQC30) and the Functional Assessment of Cancer Therapy General (FACT-G) [12, 13]. Although many mapping algorithms from the two cancer-specific measures onto the EQ-5D-3L index have been developed [14-16], there are just a few onto EQ-5D-5L [17-21]. Based on our literature survey and the latest mapping algorithm database [22], we find that there are no direct mapping algorithms for EQ-5D-5L based on the Japanese value set and no indirect (response) mapping algorithms for EQ-5D-5L from EORTC QLQ-C30 and FACT-G.

The aim of the present study is to develop direct and indirect mapping algorithms from EORTC QLQ-C30 and FACT-G onto the EQ-5D-5L index using data from the Quality Of Life Mapping Algorithm for Cancer (QOLMAC) study, where EQ-5D-5L, EORTC QLQ-C30, and FACT-G data are cross-sectionally obtained from patients with cancer.

\section{Methods}

\section{Study design and patients}

We conducted the QOL-MAC study, a multicenter, cross-sectional study to develop mapping algorithms for EORTC QLQ-C30 and FACT-G onto the EQ-5D-5L index. This study was conducted in 14 hospitals (all participating hospitals are listed in Additional file 1) in Japan from November 2018 to March 2019. The target sample size (1200 patients) was not formally based on statistical considerations. This study was conducted in accordance with the Declaration of Helsinki and the study protocol was approved by each participating hospital.

We enrolled patients with locally advanced, metastatic, or recurrent cancer with the following eligibility criteria: aged 20 or above; with lung, stomach, colorectal, or breast cancer, or any other solid tumor; under drug therapy; and with an Eastern Cooperative Oncology Group (ECOG) performance status of $0-3$. We excluded those who received treatment for multiple primary tumors or who are not able to respond to the questionnaires. We recruited both outpatients and inpatients to collect a variety of data on health status that patients with cancer could experience. All enrolled patients gave written informed consent before study enrollment.

\section{Instruments}

We conducted the EQ-5D-5L assessment using the Japanese version of the EQ-5D-5L questionnaire [11, 23], which has 5 items: mobility, self-care, usual activities, pain/discomfort, and anxiety/depression. In EQ-5D-5L, patients are asked to assign a status level (from the 5 given) to each item: no problem, slight problem, moderate problem, severe problem, or extreme problem (the wording is slightly different among the five items). We converted the responses to EQ-5D-5L into the EQ-5D-5L index using the Japanese value set based on the time trade-off method [23]. The indexes of 0 and 1 represent death and full health, respectively.

We assessed HRQOL using the Japanese versions of the EORTC QLQ-C30 questionnaire (version 3) and the FACT-G questionnaire (version 4) as the source measures in mapping algorithms [12, 13]. EORTC QLQ-C30 has 30 items and the responses to those items were converted into 5 functioning subscale scores, 9 symptom subscale scores, and a global health status score. Higher scores on the functioning subscales and global health status indicate better health condition, whereas higher scores on symptom subscales indicate severer symptom. FACT-G has 27 items and the responses to those were converted into 4 well-being subscale scores. Higher scores on the 4 well-being subscales indicate better health condition.

We asked the participants to complete a combined questionnaire of the three instruments. In addition to the three instruments, we collected data on patients' demographic and clinical characteristics.

\section{Statistical analysis}

We defined the analysis population for EORTC QLQC30 as eligible patients having both EQ-5D-5L index and all 15 subscale scores and for FACT-G as eligible patients having both EQ-5D-5L index and all 4 subscale scores. We summarized the patient characteristics and responses to EQ-5D-5L, EORTC QLQ-C30, and FACT-G 
in each analysis population. As a preliminary assessment of the conceptual overlap of the two source measures to EQ-5D-5L, we calculated Spearman's rank correlation coefficients between the subscale scores of the two source measures and the responses to the five items in EQ-5D-5L.

We developed the mapping algorithms for each source measure using 7 regression methods. Based on qualitative and quantitative assessments of the conceptual overlap between the source and target measures, all 5 functioning subscales, global health status, and two symptom subscales (fatigue and pain) were selected as initial candidate variables for direct mapping of EORTC QLQ-C30, and all 4 well-being subscales were selected as initial candidate variables for direct mapping of FACTG. For indirect mapping, we selected subscales that had an absolute rank correlation of $\geq 0.4$ (EORTC QLQ-C30) and $\geq 0.3$ (FACT-G) for each EQ-5D-5L item as initial candidate variables. Furthermore, we included age and sex into the initial candidate variables in all regression methods. We selected explanatory variables using the backward selection method, which sequentially omitted variables with the largest $P$ value $>0.15$. This $P$ value criterion approximately corresponds to the backward selection based on the Akaike information criterion [24]. No higher-order terms or interaction terms were considered.

The seven regression methods were linear regression, beta regression [25], tweedie regression [26], tobit regression, two-part linear regression, two-part beta regression [27], and ordinal logistic regression. All the regression methods except ordinal logistic regression were directly applied to the EQ-5D-5L index, whereas ordinal logistic regression was applied to each EQ-5D-5L item and used to develop the indirect mapping algorithms. In beta regression, we transformed the EQ-5D-5L index to \{observed index $-(-0.025)\} /\{1-(-0.025)\} \quad(-0.025$ is the lowest index in the Japanese value set) [25]. In tweedie regression, we transformed the EQ-5D-5L index into disutility from full health (i.e., $1-$ observed index). In tobit regression, we set the lower and upper bounds of -0.025 and 1, respectively. In two-part regression methods, we predicted full health using logistic regression. In two-part beta regression, we transformed the EQ-5D-5L index to \{observed index $-(-0.025)\} /\{0.895-(-0.025)\} \quad(0.895$ is the second largest index in the Japanese value set). In beta and two-part beta regressions, we added 0.005 and subtracted 0.005 at the lower and upper bounds, respectively [28]. We calculated the predicted EQ-5D-5L index as an expected value provided by the fitted models. For ordinal logistic regression, the predicted EQ-5D-5L index was calculated as 1 minus the sum of disutilities of the 5 levels weighted by the predicted probabilities over the 5 items.
We first evaluated the performance of our mapping algorithms based on root mean squared error (RMSE), mean absolute error (MAE), and Pearson's correlation coefficient between the observed and predicted EQ5D-5L indexes. These measures were calculated for the whole sample and ninefold cross validation. In the crossvalidation, we randomly divided the whole sample into 9 subsamples (approximately 100 patients in each subsample); repeatedly conducted variable selection in 8 subsamples and calculated the performance measures for the remaining subsample; and averaged them in subsamples to compute overfitting-corrected performance measures.

After selecting the mapping algorithms with a good predictive performance in terms of the above three measures, we checked the selected mapping algorithms in terms of face validity. We eliminated any explanatory variables that had regression coefficients with a sign that was the opposite of what was anticipated and $P>0.05$, and reestimated the regression models to obtain the final mapping algorithms. We simulated the EQ-5D-5L index from the selected final mapping algorithms and compared the mean observed and simulated EQ-5D-5L indexes in various subgroups. Furthermore, we plotted the cumulative distribution functions of observed and simulated EQ5D-5L indexes.

All statistical analyses were conducted using Base SAS, SAS/STAT, or SAS/ETS software, Version 9.4 of the SAS System for Windows.

\section{Results}

\section{Patients and descriptive data}

A total of 1031 patients were enrolled into the QOLMAC study. Of the 1029 eligible patients, 903 and 908 patients were included in the EORTC QLQ-C30 analysis population and FACT-G analysis population, respectively. Table 1 shows patients' characteristics in the two analysis populations. Lung cancer and colorectal cancer were two major tumor types; $21 \%$ of the patients were hospitalized; and $65 \%$ were receiving chemotherapy.

Table 2 shows the distributions of the EQ-5D-5L index and subscale scores of EORTC QLQ-C30 and FACTG. The mean EQ-5D-5L index was 0.781 in both analysis populations. We provided the data on the response to each EQ-5D-5L item in Additional file 1: Table S3. The correlations between the subscale scores of the two source measures and the responses to the five items in EQ-5D-5L are reported in Additional file 1: Table S4. All functioning subscales except cognitive functioning, global health status, fatigue, pain, and appetite loss in EORTC QLQ-C30 had an absolute rank correlation coefficient of $\geq 0.4$ in at least one item, whereas physical wellbeing, emotional well-being, and functional well-being 
Table 1 Patient characteristics in the two analysis populations

\begin{tabular}{|c|c|c|}
\hline Characteristic & $\begin{array}{l}\text { EORTC QLQ-C30 } \\
\mathrm{N}=903\end{array}$ & $\begin{array}{l}\text { FACT-G } \\
N=908\end{array}$ \\
\hline Age (years) & $68(58-74)$ & $68(58-74)$ \\
\hline \multicolumn{3}{|l|}{ Sex } \\
\hline Male & $489(54.2)$ & $489(53.9)$ \\
\hline Female & $414(45.8)$ & $419(46.1)$ \\
\hline \multicolumn{3}{|l|}{ Hospitalization } \\
\hline Yes & $186(20.6)$ & $180(20.8)$ \\
\hline No & $717(79.4)$ & $719(79.2)$ \\
\hline \multicolumn{3}{|l|}{ ECOG performance status } \\
\hline 0 & $451(49.9)$ & $447(49.2)$ \\
\hline 1 & $368(40.8)$ & $377(41.5)$ \\
\hline 2 & $63(7.0)$ & $64(7.0)$ \\
\hline 3 & $20(2.2)$ & $19(2.1)$ \\
\hline Unknown $(0,1,2$, or 3$)$ & $1(0.1)$ & $1(0.1)$ \\
\hline \multicolumn{3}{|l|}{ Tumor type } \\
\hline Lung cancer & $317(35.1)$ & $312(34.4)$ \\
\hline Stomach cancer & $65(7.2)$ & $64(7.0)$ \\
\hline Colorectal cancer & $222(24.6)$ & $226(24.9)$ \\
\hline Breast cancer & $113(12.5)$ & $116(12.8)$ \\
\hline Other solid tumors & $186(20.6)$ & $190(20.9)$ \\
\hline \multicolumn{3}{|l|}{ Stage at diagnosis } \\
\hline । & $49(5.4)$ & $48(5.3)$ \\
\hline$\|$ & $70(7.8)$ & $70(7.7)$ \\
\hline III & $184(20.4)$ & $185(20.4)$ \\
\hline IV & $588(65.1)$ & $593(65.3)$ \\
\hline Unknown & $12(1.3)$ & $12(1.3)$ \\
\hline \multicolumn{3}{|l|}{ Site of metastasis or recurrence ${ }^{a}$} \\
\hline None & $77(8.5)$ & $81(8.9)$ \\
\hline Liver & $198(21.9)$ & $204(22.5)$ \\
\hline Lung & $285(31.6)$ & $280(30.8)$ \\
\hline Bone & $160(17.7)$ & $165(18.2)$ \\
\hline Brain & $98(10.9)$ & $98(10.8)$ \\
\hline Lymph nodes & $361(40.0)$ & $361(39.8)$ \\
\hline Others & $225(24.9)$ & $227(25.0)$ \\
\hline \multicolumn{3}{|l|}{ History of surgery } \\
\hline Yes & $480(53.2)$ & $483(53.2)$ \\
\hline No & $422(46.7)$ & $424(46.7)$ \\
\hline Unknown & $1(0.1)$ & $1(0.1)$ \\
\hline \multicolumn{3}{|l|}{ Type of treatment ${ }^{a}$} \\
\hline Chemotherapy & $582(64.5)$ & $590(65.0)$ \\
\hline Endocrine therapy & $65(7.2)$ & $66(7.3)$ \\
\hline Molecular targeted therapy & $159(17.6)$ & $158(17.4)$ \\
\hline Immunotherapy & $108(12.0)$ & $104(11.5)$ \\
\hline Palliative therapy & $35(3.9)$ & $37(4.1)$ \\
\hline Others & $8(0.9)$ & $8(0.9)$ \\
\hline
\end{tabular}

Median (IQR) is reported for age, whereas number (\%) is reported for other characteristics

EORTC QLQ-C30, European Organization for Research and Treatment of Cancer Quality of Life Questionnaire Core 30; FACT-G, Functional Assessment of Cancer Therapy General; ECOG, Eastern Cooperative Oncology Group

${ }^{\text {a }}$ Multiple choices are allowed subscales in FACT-G had an absolute rank correlation coefficient of $\geq 0.3$ in at least one item.

\section{Model selection and selected models}

Table 3 shows the predictive performance of the fitted models. For EORTC QLQ-C30, two-part beta regression provided the best model in all three measures for the whole sample, whereas linear regression provided the best model in all three measures for cross-validation. The difference in the predictive performance was marginal between linear regression and two-part beta regression in the whole sample and cross-validation. Ordinal logistic regression had a performance that was comparable to these models for the whole sample and cross-validation. For FACT-G, two-part beta regression and ordinal logistic regression provided the best model in all three measures for the whole sample, whereas ordinal logistic regression provided the best model in all three measures for cross-validation. After inspecting face validity, the emotional well-being subscale was eliminated from the ordinal logistic regression for usual activities in FACT-G analysis. The three performance measures in whole sample after elimination of the emotional well-being were the same in the display digits as in Table 3.

Figure 1 depicts the mean EQ-5D-5L index simulated from the best-performed mapping algorithms against the observed mean EQ-5D-5L in various subgroups. The three mapping algorithms (linear, two-part beta, and ordinal logistic regression) for EORTC QLQ-C30 were well calibrated except for the subgroup with the highest global health status score, whereas the two mapping algorithms (two-part beta and ordinal logistic regression) for FACT-G provided an overestimated mean EQ-5D-5L index in the subgroups with an ECOG performance status of 2 and 3. Figure 2 shows the cumulative distribution functions of the observed and simulated EQ-5D-5L. The mapping algorithms based on two-part beta regression predicted more EQ-5D-5L index below 0.6 and less EQ5D-5L index between 0.6 to 0.9 than the observed EQ$5 \mathrm{D}-5 \mathrm{~L}$ data. The mapping algorithms based on ordinal logistic regression provided a smaller proportion of full health than the observed EQ-5D-5L data. These features were applicable to both EORTC QLQ-C30 and FACTG. For EORTC QLQ-C30, the mapping algorithm based on linear regression provided a larger proportion of full health than the true EQ-5D-5L data.

Based on the above evaluations, we recommend twopart beta regression for direct mapping algorithms and ordinal logistic regression for indirect mapping algorithms for both EORTC QLQ-C30 and FACT-G. Liner regression for EORTC QLQ-C30 is not recommended due to overestimation of the proportion of full health described in Fig. 2. Table 4 shows the estimated 
Table 2 Distributions of the EQ-5D-5L index and subscale scores of EORTC QLQ-C30 and FACT-G

\begin{tabular}{|c|c|c|c|c|c|c|c|}
\hline & Minimum & $5 \%$ & $25 \%$ & Median & $75 \%$ & $95 \%$ & Maximum \\
\hline \multicolumn{8}{|l|}{ EORTC QLQ-C30 analysis } \\
\hline EQ-5D-5L index (Japan) & -0.025 & 0.437 & 0.691 & 0.823 & 0.895 & 1 & 1 \\
\hline \multicolumn{8}{|l|}{ EORTC QLQ-C30 } \\
\hline Physical functioning & 0 & 33.3 & 66.7 & 86.7 & 93.3 & 100 & 100 \\
\hline Role functioning & 0 & 0 & 66.7 & 83.3 & 100 & 100 & 100 \\
\hline Emotional functioning & 0 & 44.4 & 75 & 83.3 & 100 & 100 & 100 \\
\hline Cognitive functioning & 0 & 33.3 & 66.7 & 83.3 & 100 & 100 & 100 \\
\hline Social functioning & 0 & 33.3 & 66.7 & 83.3 & 100 & 100 & 100 \\
\hline Global health status & 0 & 16.7 & 50 & 66.7 & 83.3 & 100 & 100 \\
\hline Fatigue & 0 & 0 & 22.2 & 33.3 & 55.6 & 77.8 & 100 \\
\hline Nausea and vomiting & 0 & 0 & 0 & 0 & 16.7 & 33.3 & 100 \\
\hline Pain & 0 & 0 & 0 & 16.7 & 33.3 & 66.7 & 100 \\
\hline Dyspnea & 0 & 0 & 0 & 33.3 & 33.3 & 100 & 100 \\
\hline Insomnia & 0 & 0 & 0 & 33.3 & 33.3 & 66.7 & 100 \\
\hline Appetite loss & 0 & 0 & 0 & 0 & 33.3 & 100 & 100 \\
\hline Constipation & 0 & 0 & 0 & 33.3 & 33.3 & 66.7 & 100 \\
\hline Diarrhea & 0 & 0 & 0 & 0 & 33.3 & 66.7 & 100 \\
\hline Financial difficulties & 0 & 0 & 0 & 33.3 & 33.3 & 100 & 100 \\
\hline \multicolumn{8}{|l|}{ FACT-G analysis } \\
\hline EQ-5D-5L index (Japan) & -0.025 & 0.436 & 0.691 & 0.823 & 0.895 & 1 & 1 \\
\hline \multicolumn{8}{|l|}{ FACT-G } \\
\hline Physical well-being & 1 & 9 & 16 & 22 & 25 & 28 & 28 \\
\hline Social/family well-being & 0 & 5 & 14 & 18 & 22 & 26.8 & 28 \\
\hline Emotional well-being & 0 & 7 & 13 & 17 & 20 & 23 & 24 \\
\hline Functional well-being & 0 & 6 & 13 & 17 & 22 & 27 & 28 \\
\hline
\end{tabular}

EORTC QLQ-C30, European Organization for Research and Treatment of Cancer Quality of Life Questionnaire Core 30; FACT-G, Functional Assessment of Cancer Therapy General

regression coefficients. Uncertainty in the estimated regression coefficients is provided in Additional file 1: Tables S5-S8 in the form of variance covariance matrix. We also provided the detailed calculation of the EQ5D-5L index from these mapping algorithms in the Additional file 1.

\section{Discussion}

In the present study, we developed direct and indirect (response) algorithms that map two common cancerspecific HRQOL measures, EORTC QLQ-C30 and FACT-G, onto the EQ-5D-5L index. The recommended direct mapping algorithms for both EORTC QLQ-C30 and FACT-G are based on two-part beta regression. These direct algorithms are suitable for generating the EQ-5D-5L index based on the Japanese value set. Conversely, the recommended indirect mapping algorithms for EORTC QLQ-C30 and FACT-G are based on ordinal logistic regression and had a predictive performance that is comparable to the recommended direct mapping algorithms. These indirect mapping algorithms can generate the EQ-5D-5L index based on a value set of any country.

For EORTC QLQ-C30, several mapping algorithms onto EQ-5D-5L index were developed. The recommended direct mapping algorithm in this study has a similar predictive performance to the largest study by Lamu et al. [18], although a direct comparison is not feasible due to the different values sets used. The best model for EQ-5D-5L in Lamu et al. was derived from two-part beta regression [18], which is the same in our study. Although Lamu et al. failed to develop indirect mapping algorithms with a good predictive performance [18], we developed indirect mapping algorithms with a predictive performance that is comparable to the direct mapping algorithms using ordinal logistic regression. Our indirect mapping algorithm can be used for any value set. Our mapping algorithms have a better predictive performance than those developed by other studies, which focus on a relatively small sample of patients with a certain cancer type $[19,20]$. 
Table 3 Predictive performance of each mapping algorithm

\begin{tabular}{|c|c|c|c|c|c|c|}
\hline & \multicolumn{3}{|c|}{ Whole sample } & \multicolumn{3}{|c|}{ ninefold cross-validation } \\
\hline & RMSE & MAE & $\rho$ & RMSE & MAE & $\rho$ \\
\hline \multicolumn{7}{|l|}{ EORTC QLQ-C30 } \\
\hline Linear & 0.099 & 0.075 & 0.838 & 0.100 & 0.076 & 0.833 \\
\hline Beta & 0.103 & 0.081 & 0.825 & 0.105 & 0.081 & 0.817 \\
\hline Tweedie & 0.110 & 0.084 & 0.803 & 0.114 & 0.086 & 0.799 \\
\hline Tobit & 0.102 & 0.077 & 0.836 & 0.103 & 0.078 & 0.822 \\
\hline Two-part linear & 0.100 & 0.075 & 0.837 & 0.101 & 0.076 & 0.825 \\
\hline Two-part beta & 0.099 & 0.075 & 0.840 & 0.101 & 0.077 & 0.828 \\
\hline Ordinal logistic & 0.100 & 0.077 & 0.835 & 0.101 & 0.078 & 0.829 \\
\hline \multicolumn{7}{|l|}{ FACT-G } \\
\hline Linear & 0.121 & 0.090 & 0.753 & 0.121 & 0.091 & 0.744 \\
\hline Beta & 0.121 & 0.091 & 0.754 & 0.122 & 0.092 & 0.752 \\
\hline Tweedie & 0.124 & 0.092 & 0.740 & 0.124 & 0.093 & 0.740 \\
\hline Tobit & 0.123 & 0.090 & 0.754 & 0.124 & 0.091 & 0.751 \\
\hline Two-part linear & 0.122 & 0.091 & 0.749 & 0.123 & 0.092 & 0.748 \\
\hline Two-part beta & 0.119 & 0.090 & 0.760 & 0.121 & 0.091 & 0.759 \\
\hline Ordinal logistic & 0.119 & 0.090 & 0.760 & 0.120 & 0.091 & 0.764 \\
\hline
\end{tabular}

The best performances in each performance measure in each source measure are italics

RMSE, root mean squared error; MAE, mean absolute error; $\rho$, correlation coefficient; EORTC QLQ-C30, European Organization for Research and Treatment of Cancer Quality of Life Questionnaire Core 30; FACT-G, Functional Assessment of Cancer Therapy General

One study has developed mapping algorithms for FACT-G onto the EQ-5D-5L index [21]. However, our recommended mapping algorithms had a better predictive performance than the previous algorithms. The recommended direct mapping algorithm for FACT-G in our study is based on two-part beta regression, which is true of EORTC QLQ-C30. Although our mapping algorithms yield a less accurate prediction of the EQ-5D-5L index than the current best mapping algorithm which additionally uses a breast cancer subscale (i.e., FACT-B) for patients with breast cancer [29], the algorithm using FACT-B cannot be used in patients with other cancers. No indirect mapping algorithm for FACT-G was available before the present study. Our indirect mapping algorithm can deal with any value set.

Although our mapping algorithms for FACT-G and EORTC QLQ-C30 have advantages over previous ones, they are not perfect. The mapping algorithms for FACT$G$ overestimates the mean EQ-5D-5L index in patients with an ECOG performance status of 2 and 3. This may be partly because FACT-G does not have a subscale directly relevant to pain/discomfort in EQ-5D-5L, whereas EORTC QLQ-C30 has a pain subscale. The pain subscale in EORTC QLQ-C30 has a rank correlation coefficient of $>0.7$ with pain/discomfort in EQ-5D-5L and was selected as an explanatory variable in the recommended direct and indirect mapping algorithms. For indirect mapping algorithms for both EORTC QLQ-C30 and FACT-G, the proportion of full health is underestimated. This underestimation by the indirect mapping algorithms was reported for EQ-5D-3L data in other disease areas [30, 31]. A new method to solve this problem in indirect mapping would be helpful to further improve the performances of indirect mapping algorithms.

Mapping from EORTC QLQ-C30 yielded smaller overall prediction errors than mapping from FACT-G. In addition to the pain subscale mentioned above, this difference in performance could be explained by the fact that the social well-being score in FACT-G is not correlated with any item in EQ-5D-5L. It is empirically known that the social well-being subscale in FACT-G measures an aspect of HRQOL different from that measured by the social functioning subscale in EORTC QLQ-C30 $[32,33]$. Besides overall prediction errors, there were two differences in the prediction performance between EORTC QLQ-C30 and FACT-G. First, FACT-G overestimated mean EQ-5D-5L index in patients with ECOG performance status 2 and 3. However, the impact of this overestimation on mapped EQ-5D-5L index may be small if mapping is applied to clinical trial data, because many patients are likely to be in relatively good condition in clinical trials. Second, the mapping algorithms for EORTC QLQ-C30 underestimated the mean EQ-5D-5L index in patients in the highest global health status group. Nevertheless, mapping algorithms for FACT-G might also underestimate the mean EQ-5D-5L index near 


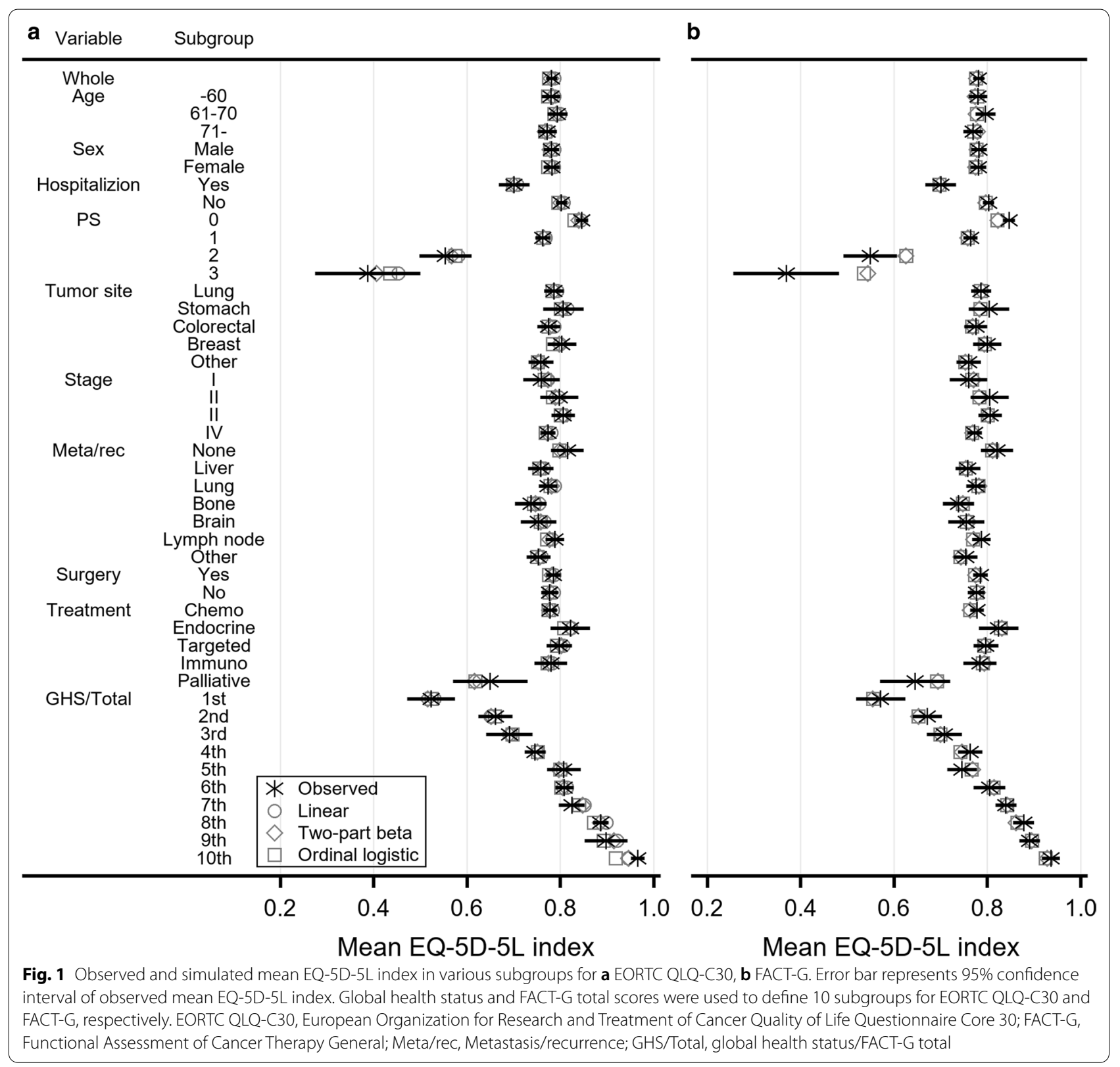

full health, because the observed mean EQ-5D-5L index in the highest FACT-G total score subgroup was lower than in the highest global health status subgroup.

To develop the mapping algorithms, we enrolled patients with 4 major cancers (lung, stomach, colorectal, and breast) and other solid tumors. Other solid tumors included prostate cancer, ovarian cancer, cervical cancer, endometrial cancer, esophageal cancer, pancreatic cancer, renal cancer, and so on. We showed that the mean EQ-5D-5L index of the 4 major cancers could be estimated accurately by the recommended mapping algorithms, although we could not assess the accuracy in patients with cancer infrequent in our data. In addition, we did not enroll patients with hematologic cancer and patients receiving adjuvant treatment after surgical resection of cancer, whereas EORTC QLQ-C30 and FACT-G can be applied to assess the HRQOL for such patients. Although compared to previous studies, we did enroll relatively diverse patients [18-21], whether the recommended mapping algorithms can be applied to these populations needs to be explored in future research.

Although we attempted to develop mapping algorithms that used item scores rather than subscale scores [34], we 

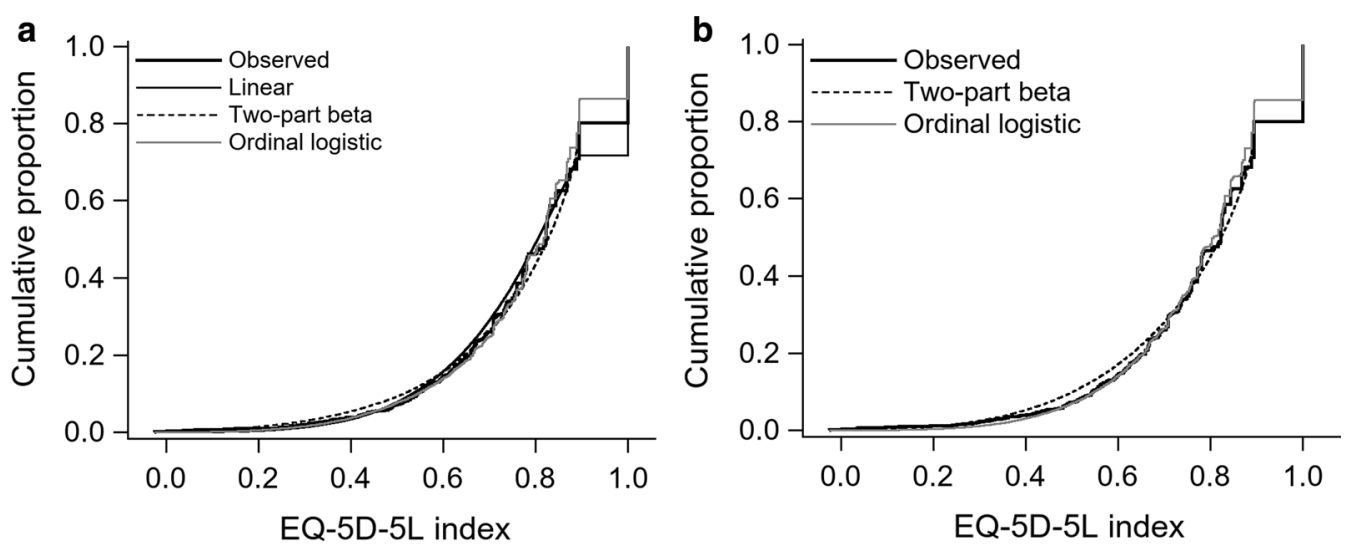

Fig. 2 Cumulative distribution functions of observed and simulated EQ-5D-5L index for a EORTC QLQ-C30, b FACT-G. EORTC QLQ-C30, European Organization for Research and Treatment of Cancer Quality of Life Questionnaire Core 30; FACT-G, Functional Assessment of Cancer Therapy General

Table 4 Regression coefficients for the recommended mapping algorithms

\begin{tabular}{|c|c|c|c|c|c|c|c|}
\hline & \multicolumn{2}{|l|}{ Two-part beta } & \multicolumn{5}{|c|}{ Ordinal logistic } \\
\hline & Logistic part & Beta part & Mobility & Self-care & Usual activities & Pain/discomfort & Anxiety/depression \\
\hline \multicolumn{8}{|l|}{ EORTC QLQ-C30 } \\
\hline Intercept 1 & -11.64060 & -1.66963 & -5.91277 & -4.24535 & -9.28712 & -0.82400 & -6.65962 \\
\hline Intercept 2 & - & - & -3.79148 & -2.56552 & -6.21474 & 2.75434 & -4.31221 \\
\hline Intercept 3 & - & - & -1.85222 & -1.23110 & -4.16801 & 5.35925 & -2.81811 \\
\hline Intercept 4 & - & - & 0.92106 & 0.17985 & -1.46187 & 7.64850 & -0.86976 \\
\hline Age & - & - & $-0.02469^{a}$ & - & - & - & $0.02119^{a}$ \\
\hline Female & - & 0.15271 & - & $0.60145^{\mathrm{a}}$ & 0.33291 & - & - \\
\hline Physical functioning & $0.03395^{\mathrm{a}}$ & $0.02475^{\mathrm{a}}$ & $0.06934^{\mathrm{a}}$ & $0.06035^{\mathrm{a}}$ & $0.04812^{\mathrm{a}}$ & - & - \\
\hline Role functioning & $0.03742^{\mathrm{a}}$ & $0.00656^{\mathrm{a}}$ & $0.01498^{\mathrm{a}}$ & $0.01912^{\mathrm{a}}$ & $0.03914^{\mathrm{a}}$ & - & - \\
\hline Emotional functioning & $0.02694^{\mathrm{a}}$ & $0.00540^{\mathrm{a}}$ & - & - & - & - & $0.06947^{a}$ \\
\hline Cognitive functioning & - & - & - & - & - & - & - \\
\hline Social functioning & - & - & - & - & 0.00770 & - & - \\
\hline Global health status & $0.03182^{\mathrm{a}}$ & $0.01167^{\mathrm{a}}$ & $0.01767^{a}$ & - & $0.02419^{a}$ & $0.02343^{\mathrm{a}}$ & - \\
\hline Fatigue & - & - & - & - & - & - & - \\
\hline Pain & $-0.06337^{a}$ & - & - & - & - & $-0.07683^{\mathrm{a}}$ & - \\
\hline Scale parameter & - & 8.09789 & - & - & - & - & - \\
\hline \multicolumn{8}{|l|}{ FACT-G } \\
\hline Intercept 1 & -11.54143 & -0.65502 & -1.86481 & -0.47810 & -5.51844 & -5.90937 & -6.77040 \\
\hline Intercept 2 & - & - & -0.17843 & 0.85700 & -3.04179 & -3.06146 & -4.14108 \\
\hline Intercept 3 & - & - & 1.19513 & 1.83808 & -1.60687 & -1.28905 & -2.67943 \\
\hline Intercept 4 & - & - & 3.02125 & 2.87152 & 0.22225 & 0.45253 & -0.88517 \\
\hline Age & - & $-0.01111^{\mathrm{a}}$ & $-0.04819^{a}$ & $-0.04255^{a}$ & -0.02439 & - & 0.01664 \\
\hline Female & - & - & - & - & - & - & - \\
\hline Physical well-being & $0.34876^{\mathrm{a}}$ & $0.09845^{\mathrm{a}}$ & $0.21038^{a}$ & $0.19205^{a}$ & $0.26197^{a}$ & $0.24215^{\mathrm{a}}$ & $0.04892^{\mathrm{a}}$ \\
\hline Social well-being & - & - & - & - & - & - & - \\
\hline Emotional well-being & 0.06963 & $0.03355^{\mathrm{a}}$ & - & - & - & - & $0.26968^{\mathrm{a}}$ \\
\hline Functional well-being & $0.04585^{\mathrm{a}}$ & $0.03922^{\mathrm{a}}$ & $0.05296^{\mathrm{a}}$ & $0.07714^{\mathrm{a}}$ & $0.08561^{\mathrm{a}}$ & 0.01740 & $0.04819^{a}$ \\
\hline Scale parameter & - & 6.05147 & - & - & - & - & - \\
\hline
\end{tabular}

EORTC QLQ-C30, European Organization for Research and Treatment of Cancer Quality of Life Questionnaire Core 30; FACT-G, Functional Assessment of Cancer Therapy General

a $P<0.01$ (not applicable to intercepts and scale parameter) 
did not report the detailed results because of two considerations. First, the items models for EORTC QLQ-C30 improved the predictive performance for the whole sample but did not for the cross-validated versions, suggesting an overfitting. Second, the item models for FACT-G did improve the cross-validated predictive performance too but had low face validity (i.e., many estimated regression coefficients had signs opposite to what was anticipated). Despite their improved performance, the item models for FACT-G had a lower predictive performance than the recommended subscale models for EORTC QLQ-C30. From the statistical viewpoint, subscale models stabilize estimation results by assuming the same regression coefficient for items in each subscale, since a subscale score in EORTC QLQ-C30 and FACT-G is essentially the sum of the item scores in the corresponding subscale with the same weight. Good reliability (e.g., a high Cronbach's $\alpha$ ) of the two cancer-specific HRQOL measures suggests that the item scores in a subscale are highly correlated and can, thus, induce multicollinearity, which is likely to result in low face validity.

Several limitations should be taken into consideration when interpreting the results of this study. First, we used only the Japanese value set for EQ-5D-5L; thus, our direct mapping algorithms may not be suitable for cost-effectiveness analyses that use any other value set. To avoid this, we developed indirect mapping algorithms that can produce the EQ-5D-5L index based on any value set, although their performance was investigated only for the Japanese value set. This limitation is applicable to comparison of the prediction performance between EORTC QLQ-C30 and FACT-G. Second, we did not conduct external validation using external data. Third, we did not consider higher-order terms or interaction terms, which could have further improved the mapping algorithms. However, the need for higher-order and interaction terms depends on the scale of outcome. Since we used identity, log, and logit link functions while developing the mapping algorithms, some impact of higher-order and interaction terms would have been considered. Fourth, we did not apply adjusted limited dependent mixture models that show good performance for EQ-5D-3L data from patients with cancer $[35,36]$. The usefulness of this method is unclear for EQ-5D-5L data that does not show multimodality and it should be investigated in future studies.

\section{Conclusions}

Using data obtained from patients receiving drug therapy for cancer, we developed direct and indirect (response) mapping algorithms for EORTC QLQ-C30 and FACT-G onto the EQ-5D-5L index. In cost-effectiveness analyses, the developed mapping algorithms can provide the
EQ-5D-5L index whose performance would be as good or better than that of previous algorithms.

\section{Supplementary information}

Supplementary information accompanies this paper at https://doi. org/10.1186/s12955-020-01611-w.

Additional file 1. Calculation of the EQ-5D-5L index from the recommended mapping algorithms, supplementary tables, and list of hospitals participating in the QOL-MAC study.

\section{Abbreviations \\ EORTC QLQ-C30: European Organization for Research and Treatment of Cancer Quality of Life Questionnaire Core 30; FACT-G: Functional Assessment of Cancer Therapy General; RMSE: Root mean squared error; QALY: Quality- adjusted life year; HRQOL: Health-related quality of life; QOL-MAC: Quality of life mapping algorithm for cancer; ECOG: Eastern cooperative oncology group; MAE: Mean absolute error; PHRF: Public Health Research Foundation.}

\section{Acknowledgements}

We thank the patients who participated in the QOL-MAC study. This study was supported by the Comprehensive Support Project for Oncology Research of PHRF. We are grateful to the investigators and staff who dedicated their time to this study (all participating hospitals are listed in the Additional file 1). We thank Qol Co., Ltd. for their support in recruiting patients.

\section{Authors' contributions}

All authors contributed to the conception or design of the study. NT contributed to the acquisition of data. YH contributed to the analysis of data. All authors contributed to the interpretation of data. YH wrote the first draft of the manuscript and all authors revised it critically. All authors read and approved the final manuscript.

\section{Funding}

The QOL-MAC study was sponsored by the Public Health Research Foundation (PHRF). The research fund was provided to PHRF by Center for Outcomes Research and Economic Evaluation for Health, National Institute of Public Health under the study contract. This work was partially supported under Grant 19K24193 from the Japan Society for the Promotion of Science, KAKENHI.

\section{Availability of data and materials}

The data that support the findings of this study are available from Center for Outcomes Research and Economic Evaluation for Health, National Institute of Public Health, but restrictions apply to the availability of these data, which were used under license for the current study, and so are not publicly available.

\section{Ethics approval and consent to participate}

The study protocol was approved by each participating hospital. All enrolled patients gave written informed consent before study enrollment.

\section{Consent for publication}

No details of individual participants are described in this paper.

\section{Competing interests}

All authors have declared no conflict of interest.

\section{Author details}

${ }_{1}^{1}$ Department of Biostatistics, Division of Health Sciences and Nursing, The University of Tokyo, 7-3-1, Hongo, Bunkyo-ku, Tokyo 113-0033, Japan. ${ }^{2}$ Center for Outcomes Research and Economic Evaluation for Health, National Institute of Public Health, Wako, Japan. ${ }^{3}$ Breast and Endocrine Surgery Department, Okayama University Hospital, Okayama, Japan. ${ }^{4}$ Clinical Research Promotion Center, The University of Tokyo Hospital, Tokyo, Japan. ${ }^{5}$ Center for Health Economics and QOL Research, Niigata University of Health and Welfare, 
Niigata, Japan. ${ }^{6}$ Department of Biomedical Sciences, College of Life Sciences, Ritsumeikan University, Kusatsu, Japan.

Received: 19 July 2020 Accepted: 26 October 2020

Published online: 03 November 2020

\section{References}

1. Bray F, Ferlay J, Soerjomataram I, Siegel RL, Torre LA, Jemal A. Global cancer statistics 2018: GLOBOCAN estimates of incidence and mortality worldwide for 36 cancers in 185 countries. CA Cancer J Clin. 2018:68:394-424.

2. Yu PP, Eton O, Garrison LP. Challenges in assessing the clinical utility and economic value of immune checkpoint inhibitor therapies of Cancer. J Immunother Cancer. 2019;7:1-6.

3. Verma V, Sprave T, Haque W, Simone CB, Chang JY, Welsh JW, et al. A systematic review of the cost and cost-effectiveness studies of immune checkpoint inhibitors. J Immunother Cancer. 2018;6:1-15.

4. Weinstein M, Torrance G, Mcguire A. QALYs: The Basics. Value Health. 2009;12:S5-9.

5. Brooks R, De Charro F. EuroQol: the current state of play. Health Policy. 1996;37:53-72.

6. The National Institute for Health and Care. Guide to the methods of technology appraisal 2013. Available from: https://www.nice.org.uk/process/ pmg9/resources/guide-to-the-methods-of-technology-appraisal-2013pdf-2007975843781\%0A. https://www.nice.org.uk/process/pmg9/chapt er/foreword.

7. Hasegawa M, Komoto S, Shiroiwa T, Fukuda T. Formal implementation of cost-effectiveness evaluations in Japan: a unique health technology assessment system. Value Health. 2019;23:43-51.

8. Center for Outcomes Research and Economic Evaluation for Health National Institute of Public Health (C2H). Guideline for preparing costeffectiveness evaluation to the Central Social Insurance Medical Council. Version 2.0 (Internet). Available from: https://c2h.niph.go.jp/tools/guide line/guideline_en.pdf.

9. Janssen MF, Bonsel GJ, Luo N. Is EQ-5D-5L better than EQ-5D-3L? A headto-head comparison of descriptive systems and value sets from seven countries. Pharmacoeconomics. 2018;36:675-97.

10. Selivanova A, Buskens E, Krabbe PFM. Head-to-head comparison of EQ-5D-3L and EQ-5D-5L health values. Pharmacoeconomics. 2018;36:715-25.

11. Herdman M, Gudex C, Lloyd A, Janssen M, Kind P, Parkin D, et al. Development and preliminary testing of the new five-level version of EQ-5D (EQ-5D-5L). Qual Life Res. 2011;20:1727-36.

12. Aaronson NK, Ahmedzai S, Bergman B, Bullinger M, Cull A, Duez NJ, et al. The European Organization for Research and Treatment of Cancer QLQC30: a quality-of-life instrument for use in international clinical trials in oncology. J Natl Cancer Inst. 1993;85:365-76.

13. Cella DF, Tulsky DS, Gray G, Sarafian B, Linn E, Bonomi A, et al. The functional assessment of cancer therapy scale: development and validation of the general measure. J Clin Oncol. 1993;11:570-9.

14. Doble B, Lorgelly P. Mapping the EORTC QLQ-C30 onto the EQ-5D-3L: assessing the external validity of existing mapping algorithms. Qual Life Res. 2016;25:891-911.

15. Teckle P, McTaggart-Cowan H, Van der Hoek K, Chia S, Melosky B, Gelmon $K$, et al. Mapping the FACT-G cancer-specific quality of life instrument to the EQ-5D and SF-6D. Health Qual Life Outcomes. 2013;11:1-10.

16. Young TA, Mukuria C, Rowen D, Brazier JE, Longworth L. Mapping functions in health-related quality of life: mapping from two cancer-specific health-related quality-of-life instruments to EQ-5D-3L. Med Decis Making. 2015;35:912-26.

17. Khan I, Morris S, Pashayan N, Matata B, Bashir Z, Maguirre J. Comparing the mapping between EQ-5D-5L, EQ-5D-3L and the EORTC-QLQ-C30 in non-small cell lung cancer patients. Health Qual Life Outcomes. 2016;14:1-15.

18. Lamu AN, Olsen JA. Testing alternative regression models to predict utilities: mapping the QLQ-C30 onto the EQ-5D-5L and the SF-6D. Qual Life Res. 2018;27:2823-39.
19. Ameri H, Yousefi M, Yaseri M, Nahvijou A, Arab M, Sari AA. Mapping the cancer-specific QLQ-C30 onto the generic EQ-5D-5L and SF-6D in colorectal cancer patients. Expert Rev Pharmacoecon Outcomes Res. 2019;19:89-96.

20. Ameri H, Yousefi M, Yaseri M, Nahvijou A, Arab M, Sari AA. Mapping EORTC-QLQ-C30 and QLQ-CR29 onto EQ-5D-5L in colorectal cancer patients. J Gastrointest Cancer. 2020;51:196-203.

21. Meregaglia M, Borsoi L, Cairns J, Tarricone R. Mapping health-related quality of life scores from FACT-G, FAACT, and FACIT-F onto preference-based EQ-5D-5L utilities in non-small cell lung cancer cachexia. Eur J Heal Econ. 2019;20:181-93.

22. Dakin H, Abel L, Burns R, Yang Y. Review and critical appraisal of studies mapping from quality of life or clinical measures to EQ-5D: An online database and application of the MAPS statement. Health Qual Life Outcomes. Health and Quality of Life Outcomes; 2018;16:1-9.

23. Shiroiwa T, Ikeda S, Noto S, Igarashi A, Fukuda T, Saito S, et al. Comparison of value set based on DCE and/or TTO data: scoring for EQ-5D-5L health states in Japan. Value Health. 2016;19:648-54.

24. Steyerberg EW. Clinical prediction models. 2nd ed. New York: Springer; 2019.

25. Khan I, Morris S. A non-linear beta-binomial regression model for mapping EORTC QLQ- C30 to the EQ-5D-3L in lung cancer patients: a comparison with existing approaches. Health Qual Life Outcomes. 2014;12:1-16

26. Dunn PK, Smyth GK. Series evaluation of Tweedie exponential dispersion model densities. Stat Comput. 2005;15:267-80.

27. Ospina R, Ferrari SLP. A general class of zero-or-one inflated beta regression models. Comput Stat Data Anal. 2012;56:1609-23.

28. Hunger M, Döring A, Holle R. Longitudinal beta regression models for analyzing health-related quality of life scores over time. BMC Med Res Methodol. 2012;12:144.

29. Yang Q, Yu XX, Zhang W, Li H. Mapping function from FACT-B to EQ-5D-5 L using multiple modelling approaches: data from breast cancer patients in China. Health Qual Life Outcomes. 2019;17:1-12.

30. Hernández Alava M, Wailoo A, Wolfe F, Michaud K. A Comparison of direct and indirect methods for the estimation of health utilities from clinical outcomes. Med Decis Making. 2014;34:919-30.

31. Wailoo A, Hernández M, Philips C, Brophy S, Siebert S. Modeling health state utility values in ankylosing spondylitis: comparisons of direct and indirect methods. Value Health. 2015;18:425-31.

32. Luckett T, King MT, Butow PN, Oguchi M, Rankin N, Price MA, et al. Choosing between the EORTC QLQ-C30 and FACT-G for measuring healthrelated quality of life in cancer clinical research: Issues, evidence and recommendations. Ann Oncol. 2011;22:2179-90.

33. Kemmler BG, Holzner B, Kopp M, Du M, Margreiter R, Greil R, et al. Comparison of two quality-of-life instruments for cancer patients: the functional assessment of cancer therapy-general and the European Organization for Research and Treatment of Cancer Quality of Life Questionnaire-C30. J Clin Oncol. 1999;17:2932-40.

34. Wailoo AJ, Hernandez-Alava M, Manca A, Mejia A, Ray J, Crawford B, et al. Mapping to estimate health-state utility from non-preference-based outcome measures: an ISPOR good practices for outcomes research task force report. Value Health. 2017:20:18-27.

35. Hernández Alava M, Wailoo AJ, Ara R. Tails from the peak district: adjusted limited dependent variable mixture models of EQ-5D questionnaire health state utility values. Value Health. 2012;15:550-61.

36. Gray LA, Wailoo AJ, Hernandez AM. Mapping the FACT-B instrument to EQ-5D-3L in patients with breast cancer using adjusted limited dependent variable mixture models versus response mapping. Value Health. 2018:21:1399-405.

\section{Publisher's Note}

Springer Nature remains neutral with regard to jurisdictional claims in published maps and institutional affiliations. 